\title{
Laser Doppler Flowmetry versatile acquisition system with low quantization error
}

\author{
Francesco Marazzi ${ }^{1}$, Frederic Truffer $^{2}$, Martial Geiser*2 \\ ${ }^{1}$ University of Modena and Reggio Emilia, Modena, Italy \\ ${ }^{2}$ University of Applied Sciences of Western Switzerland, Sion, Switzerland
}

Received: January 12, 2018

Accepted: February 6, 2018

Online Published: February 24, 2018

DOI: $10.5430 /$ jbei.v4n1p25

URL: https://doi.org/10.5430/jbei.v4n1p25

\begin{abstract}
The Laser Doppler Flowmetry (LDF) is a non-invasive technique used to evaluate blood perfusion of various human tissues like the skin or the fundus of the eye. It is based on the scattering of light on moving red blood cells in tissue. Frequency shifted scattered light is detected and provide an electrical signal. Physical models for LDF use the DC and AC components of this signal. If AC is small relative to the DC, digitalization becomes an issue, and if more than two LDF signal acquisitions and analysis have to be done simultaneously, the device becomes expensive and bulky. We propose here a versatile and inexpensive acquisition system, which overcomes quantization errors issue by first separating DC from AC, then amplifying AC and finally recombining both signals before digitalization. We designed an analog circuit combined with a 12 bit analog-to-digital converter, a microcontroller unit and a Raspberry Pi2 (Rpi2) for the signal processing. Results are accessed remotely from the Rpi2 through HTTP protocol. Multiple systems can easily be used simultaneously for multichannel acquisitions.
\end{abstract}

Key Words: Laser Doppler Flowmetry, Microcirculation, Signal acquisition, Low quantization

\section{INTRODUCTION}

Laser Doppler Flowmetry (LDF) is a non invasive technique $^{[1-4]}$ that allows the monitoring of microvascular blood flow in clinical routines. This technique can be used in dermatology to assess the degree of skin irritability in patch test procedures, in pharmacology to study the microvascular effects of vasoactive substances and drugs, in plastic surgery, ${ }^{[5]}$ to study the changes in microvascular blood flow in diabetic patients ${ }^{[6-9]}$ or in people with peripheral vascular diseases ${ }^{[10,11]}$ of with Raynaud's phenomenon. ${ }^{[12-14]}$

LDF is also applied to the human eye to study blood flow in the optic nerve head, ${ }^{[15]}$ the retinal microcirculation ${ }^{[16]}$ and the choriocapillaris in the foveal region of the choroid, ${ }^{[17]}$ which may become important in the investigation of the pathogenesis of age-related macular degeneration. ${ }^{[18,19]}$ In the LDF technique, a coherent light is focused on a living tissue; most of the light is diffused by the static tissue and a part is reflected by the moving red blood cells (RBC) leading to Doppler shifted light ${ }^{[3,4]}$ with various frequency shifts $\Delta f_{s}$ proportional to the scatters velocity. ${ }^{[20]}$ Some of the backscattered light (frequency shifted and non-shifted) is optically collected on a detector, which produces an electrical beating signal from which information about blood perfusion can be extracted. ${ }^{[2]}$ This is done through the Doppler shift power spectrum (DSPS). ${ }^{[21]}$ The computation for the blood perfusion parameters are: 6

\footnotetext{
*Correspondence: Martial Geiser; Email: martial.geiser@hevs.ch; Address: University of Applied Sciences of Western Switzerland, Sion, Switzerland.
}

Published by Sciedu Press 
- The $D C$ value, proportional to the intensity of the detected light.

- The first moment of the DSPS ( $\mathrm{Vel}$ ), proportional to the mean velocities of the RBCs.

- The power of the DSPS, normalized with the $D C^{2}$ $(V o l)$, which is assumed to be proportional to the number of scatters within the probing volume.

To get a reliable DSPS and to reduce the variance of the measurements, ${ }^{[22]}$ it is important to keep during the recording a constant amount of backscattered light $(D C)$ and to use an analog-to-digital converter (ADC) with a high amplitude resolution (usually 12 or 16 bits). The useful information can be extracted by the part of the spectra between $30 \mathrm{~Hz}$ and $40 \mathrm{kHz}$, so that a sampling rate of at least $80 \mathrm{kHz}$ has to be used, in agreement with the Nyquist-Shannon sampling theorem. To be on the safe side, usually the sampling rate is around $120 \mathrm{kHz}$. When the $A C$ part of the signal is very small compared to the $D C$, quantification error occurs.
To reduce such error, a simple way would be to increase the amplitude resolution of the ADC. However, doing this makes the electronic board very sensitive to electronic noise. We wanted a simple design, easy to manufacture and cost effective. Thus we designed and tested a new independent single intelligent detection unit in which only the $A C$ is amplified, and an offset is added to the $D C$. The signal is analyzed by an on-board computer, in which a web server is running for further access. Such unit will do the analysis on board, simplifying the acquisition while reducing cost and separating the code for signal analysis done on the Rpi2 and that for the human interface done for a web browser. This code separation will allow further development of smart LDF systems able to do simultaneous multiple measurements.

\section{MATERIALS AND METHODS}

\subsection{Technique}

The overall principle of the LDF signal analysis is shown in Figure 1.

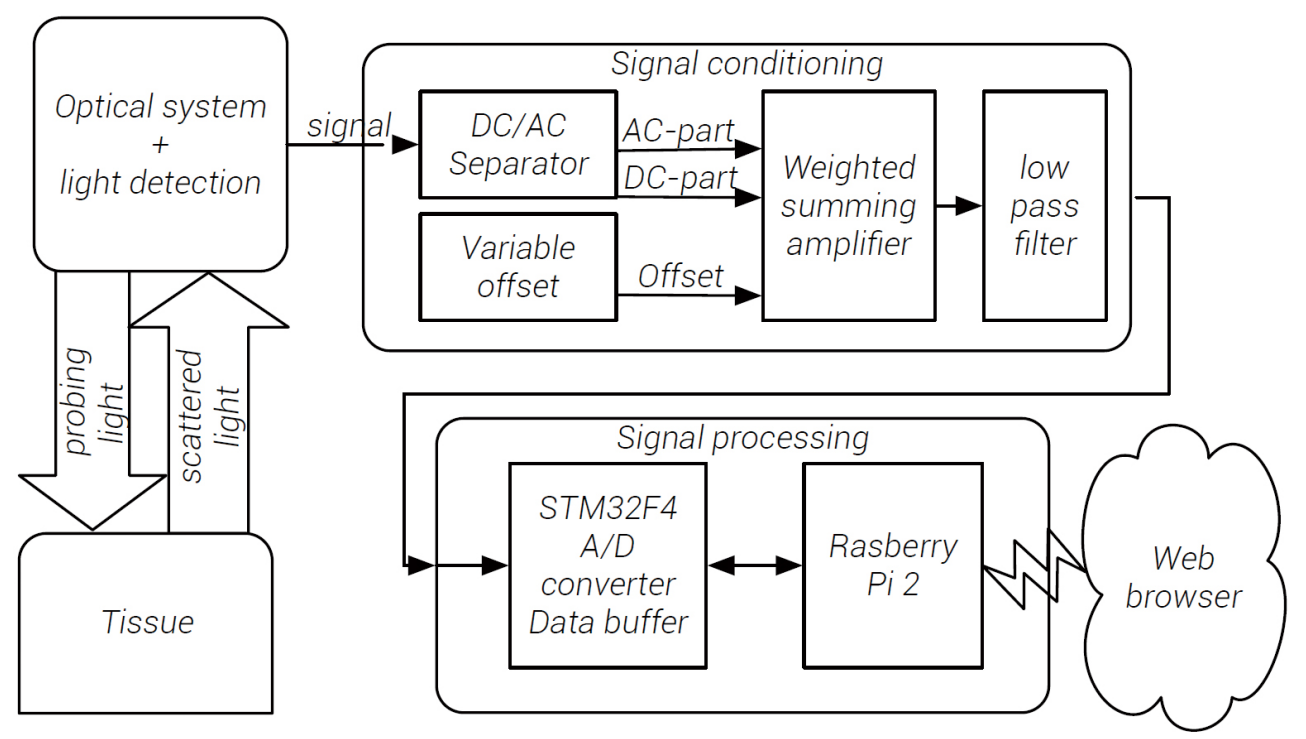

Figure 1. General view of the Laser Doppler Flowmetry acquisition system

\subsubsection{LDF set-up}

For simplicity, we assumed that a probing light hits a tissue under investigation and that an optical system collect a part of the scattered light and send it to a detector which delivers an electrical signal.

\subsubsection{Signal conditioning}

The expected DC component of the input signal is either positive $[0,1] \mathrm{V}$ or negative $[-1,0] \mathrm{V}$, depending on the detector type. The $A C$ component of the signal is expected to have an RMS in the $\mathrm{mV}$ range. Because following stages requires a positive signal, possibility of inversion was implemented. The input signal is separated into its two components $(A C$ and $D C$ ), which follow two different electrical paths. A variable $D C$ offset is also generated. Its amplitude can be controlled by using a variable resistor. The $A C, D C$ and $D C$ offset are added using a weighted sum amplifier. The $D C$ and its $D C$ offset will have a weight equal to 1 , while the weight of the $A C$ part (the gain) is set to 15 . Finally, a 10th order low-pass digital filter (LTC1569-7, Linear Technology) with a cutoff frequency of $43 \mathrm{kHz}$ is used. It is followed by 
a passive low pass filter with cutoff frequency of $100 \mathrm{kHz}$ to reduce the effect of clock feed forward. This solution has been chosen for two reasons:

- The variable offset, coupled with the amplification of the $A C$ components, allows the ADC to be used in its full range (0-3 V) independently of the input.

- It is possible to cancel the effects of the background noise by simply changing the $D C$ offset accordingly, keeping the $D C$ of the signal in the middle of the ADC input range $(1.5 \mathrm{~V})$.

\subsubsection{Signal processing}

A Microcontroller Unit (MCU, STM32F407) using Direct Memory Access, will sample the conditioned signal and store the digital converted value (12 bits) in a variable that acts as a buffer. This avoids loss of data due to the fact that the operating system of the Raspberry $\mathrm{Pi}$ is not real time. To avoid losing data at the sampling frequency used in this project $(138 \mathrm{kS} / \mathrm{s})$, the transfer between the Raspberry and the MCU starts as soon as the buffer is half filled. Starting the transmission with half buffer still empty allows us to keep storing data during the transmission, without overwriting old samples. To transfer the values at a higher rate than the sampling frequency, the samples stored in the MCU are transferred to the Raspberry Pi using the SPI channel. The Raspberry Pi then saves the values in the Comma Separated Value (CSV) file which can be locally processed or retrieved via Ethernet. The commands to start or stop the measurement are sent via Ethernet. Biomedical signal artefacts such as skin movement or eye blinks and any signal added to the RBCs Doppler shift have to be recognized by software and then removed.

This system has been used to measure the perfusion on the fundus of the eye. The filtering of each frame, if acquired on the eye of a living patient, happens in three steps: ${ }^{[23]}$

- $D C$ : Frames which $D C$ are not within $\pm 20 \%$ of the mean are discarded. This ensures a stable signal.

- Vel: Frames are discarded if the DSPS around $100 \mathrm{~Hz}$ is 10 times lower than the DSPS around $30 \mathrm{kHz}$. This ensures a meaningful Doppler signal.

- Vol: Sudden changes larger than $2 \times$ the previous value, which occurs $1 / 15$ of a second before and are due to microsaccades of the eye, were disregarded.

Those values have been chosen based on experiments.
From the digitalized recording (CSV file), further studies were done. Even though there is the possibility to process the data directly on the Raspberry, we have chosen to study the recording on an external system because visualization of the data was faster on a PC.

\subsection{Experiments}

To validate the new system, we used an existing LDF device (FloM-S). ${ }^{[23]}$ We also followed the same experimental procedure as previously described. ${ }^{[23]}$ We analyzed at the same time with both systems the electrical signal coming from the avalanche photodiode of the device. The frequency window for perfusion calculation was from $30 \mathrm{~Hz}$ to $30 \mathrm{kHz}$ and the noise level was calculated from the signal between $35 \mathrm{kHz}$ to $40 \mathrm{kHz}$. This allowed us to compare the results between the FloM-S device and the new Doppler acquisition proposed here (Rpi2-acquisition).

\subsubsection{Measurement on a rotating diffusing wheel, used as a Doppler shift generator}

The probing beam of the FloM-S device was focused on a rotating Teflon wheel $45 \mathrm{~mm}$ in diameter using a $20 \mathrm{~mm}$ focal lens. The backscattered signal exhibits a Doppler shifts which is similar to the one produced by the human eye. In that case, the mean frequency shift ( $\mathrm{Vel}$ ) is expected to be proportional to the speed of the wheel and because the probing volume does not change and the number of scatter is constant (homogeneous Teflon), $\mathrm{Vol}$ is expected to remain constant.

We recorded simultaneously with the FloM and the Rpi2 the flow parameters at 7 different wheel speeds.

\subsubsection{Measurement on the choroid of human eyes}

The following comparison complied with the tenets of the Declaration of Helsinki for human experimentation. Pupils were not dilated.

Seven healthy subjects were asked to look with one random eye a faint red light point (probing beam) for 30 seconds during which a recording was done.

\section{RESULTS}

\subsection{Measurement on a rotating diffusing wheel, used as a Doppler shift generator}

Figure 2 shows that the power spectrum obtained using a rotating diffusing wheel (artificial eye) and the one obtained on the eye of a volunteer have similar frequency shifts and same magnitude of power at frequencies larger than $500 \mathrm{~Hz}$. The frequency resolution is $8 \mathrm{~Hz}$. Mean frequencies are also comparable. 


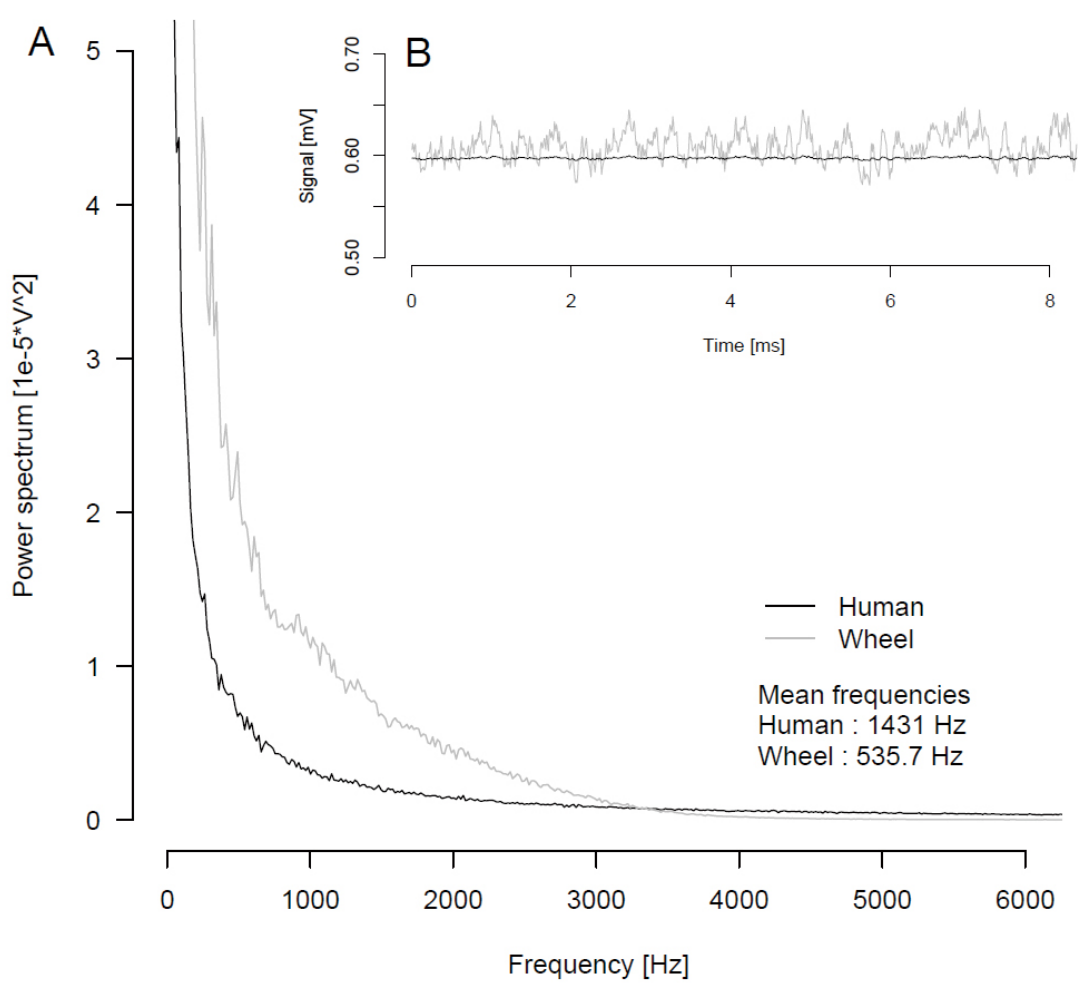

Figure 2. A: Comparison between the power spectrum obtained using a rotating wheel and a human eye as Doppler generators. B: An example of an FloM-S signal (black) and that obtained with the Rpi2 acquisition (gray).

The comparison of the results obtained with the Teflon wheel than that of the FloM-S which suggest that the quantification at different rotating speed is shown in Figure 3. From the noise is lower, assuming an electronic noise similar in both fit, the $\mathrm{Vel}$ value at zero speed of the Rpi2 data is lower cases.

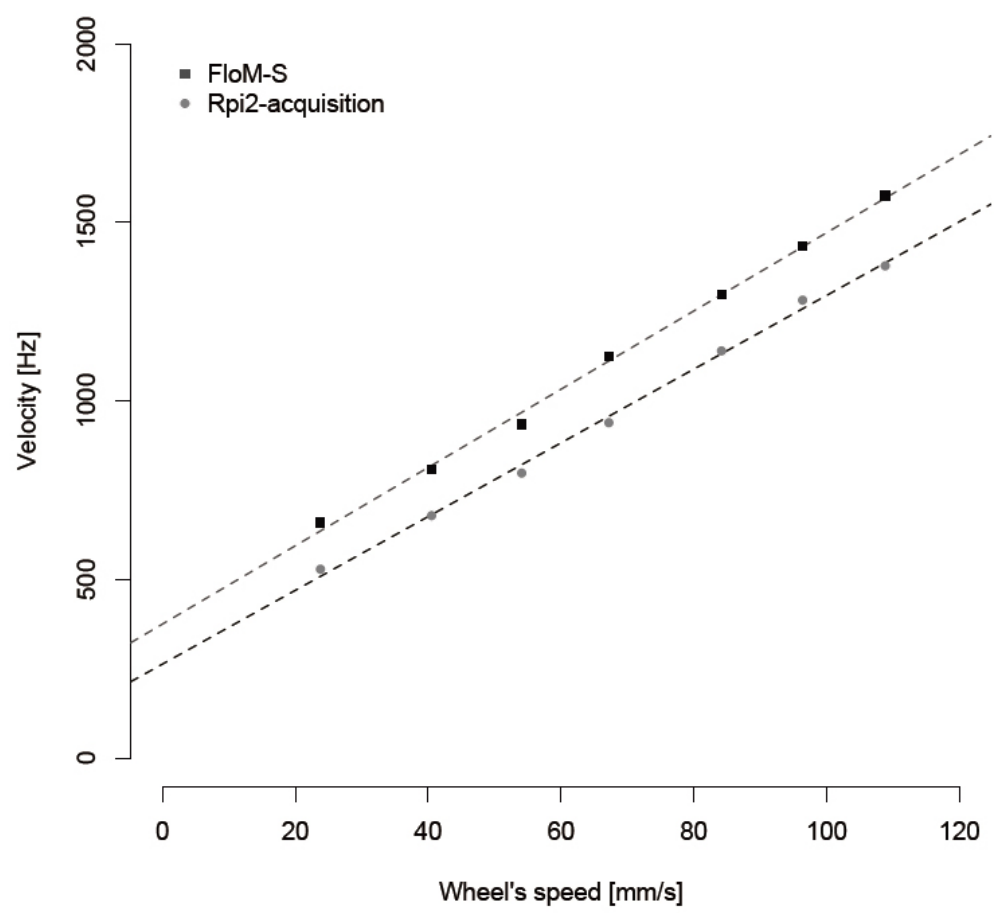

Figure 3. Velocity parameter obtained using the rotating wheel with the Rpi2 acquisition board and the FloM-S 


\subsection{Measurement on the choroid of human eyes}

The scattered light collected is composed mostly of unshifted light with a weak part Doppler shifted. In the case of the FloM-S, the ratio is about 1:500 (see Figure 2B).

For all 30 seconds recording, subjects have different perfusion parameters distribution, but the standard deviation of $V e l_{R P i}$ (mean $549.7 \mathrm{~Hz}$ ) is systematically lower than $\mathrm{Vel}_{\text {FloM-S }}$ (mean $736.3 \mathrm{~Hz}$ ) by $187 \mathrm{~Hz}$ in mean. Also the mean of the Kurtosis value for $\mathrm{Vel}_{\mathrm{FloM}-S}$ is smaller than that of $V e l_{R P i}$, this difference is statistically not significant.

Figure 4 shows the distribution of the 30 seconds measurements of the vel parameter for all subjects with the FloM$\mathrm{S}$ device and with the Rpi2-acquisition. Kurtosis tend to be larger with FloM $-S(7.9 \pm 12.3)$, which would indicate that tails are more populated than in the Rpi2 acqui- sition $(2.0 \pm 2.1)$, however this is very subject dependent. Skewness is smaller with Rpi2 acquisition ( -1.27 to 0.12$)$ than FloM-S (-1.63 to 2.68) but no statistical difference is found. However, the reduction with Rpi2-acquisition of the standard deviation of the $\mathrm{Vel}$ distribution is statistically significant $(p=.006,743 \mathrm{~Hz}$ for FloM-S to $535 \mathrm{~Hz}$ for Rpi2-acquisition).

Table 1. Statistical values for the velocity from the recordings of 19 eyes measured simultaneously with the FloM-S and the Rpi2-acquisition

\begin{tabular}{lll}
\hline & FloM-S & Rpi2 \\
\hline Min & $1,994 \mathrm{~Hz}$ & $1,331 \mathrm{~Hz}$ \\
Max & $4,122 \mathrm{~Hz}$ & $3,637 \mathrm{~Hz}$ \\
Mean \pm SD & $3,434 \pm 644 \mathrm{~Hz}$ & $2,882 \pm 715 \mathrm{~Hz}$ \\
Skewness \pm SD & $-0.458 \pm 1.104$ & $-0.556 \pm 0.465$ \\
kurtosis \pm SD & $4.577 \pm 7.923$ & $1.669 \pm 2.084$ \\
\hline
\end{tabular}

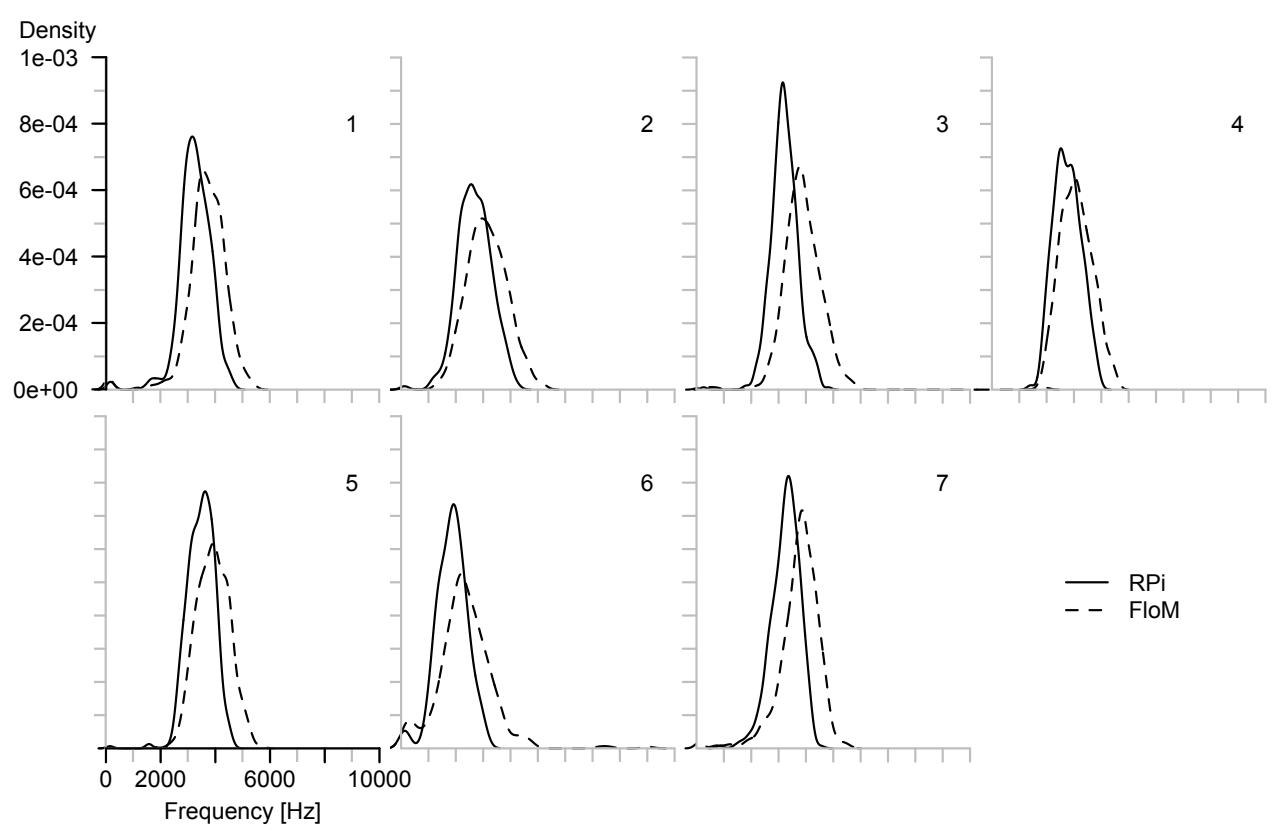

Figure 4. Density distributions versus frequency shifts of seven -30 seconds- recordings obtained with the FloM-S (dotted lines) and with the new Rpi2 device (continuous lines). All graphs have the same scales.

\section{Discussion}

The perfusion values $\mathrm{Vel}$ and $\mathrm{Vol}$ obtained with the Rpi2acquisition system have the same linear relationship with the speed of the wheel as those obtained with the FloM-S. Vel has shown a strong linear relationship with the speed of the wheel. $\mathrm{Vol}$ also increased, but very slowly and all values remained within $\pm 10 \%$ of its mean, which was also expected. The linear fitting of $\mathrm{Vel}$, in both systems, reveals a non-zero offset. There are multiple reasons behind this inconsistency:

- The presence of noise, i.e. Brownian motion, lateral movement and electrical noise.
- The parameters used for the data processing. The presence of a lower bound $(30 \mathrm{~Hz})$ removes some useful spectral components. The upper bound, on the other hand, has to be chosen carefully: If it is too low, useful signal components will be removed, if it is too high, more noise will be taken into account in the measurement.

- The spectra, obtained by measurements in the human eye, have a long tail, thus the choice of the frequency window to determine the noise floor impacts the calculation of the perfusion parameters. 
The new system yields a reduced $\mathrm{Vel}$ offset thanks to the reduction in the quantization noise and the electrical noise. The FloM-S had a laptop and its power supply close to the laser, as opposed to the new system that only uses the Raspberry, which generates less electrical noise.

For any given recording, the Rpi2-acquisition system provides measurements distribution with a lower standard deviation than that from the FloM-S. This is interpreted mainly as a lower quantification noise. The presence of a larger $\mathrm{Vel}$ offset in the old acquisition system reduces the effect of this difference in the coefficient of variation parameters.

Compared to the FloM-S acquisition system excluding the computer for the analysis, the cost the Rpi2-system has been reduced by a factor ten.

A given measurement of 30 seconds (saved in a CSV file) has been computed on both systems and the timings have been measured and compared. On the Raspberry Pi, the computation takes around 5 seconds, while on the PC (Intel
Core $17-4790 \mathrm{CPU}$ ) it takes 0.5 seconds. This shows that the Raspberry is perfectly capable of computing the data in a reasonable amount of time.

\section{Conclusion}

We showed that our approach to amplify only the AC part of the Doppler signal before digitalizing leads to less noisy signal with a smaller standard deviation and thus a higher sensitivity to physiological changes of the perfusion parameters $\mathrm{Vel}$ and $\mathrm{Vol}$. Moreover, the use of tiny computers such as Rasberry Pi, reduces the price of the Doppler signal acquisition while simplifying the programming of the user interface. Also, the reduced dimensions and power requirement of the proposed system allows an easy acquisition and processing of multiple optical input channels at the same time.

\section{ACKNOWLEDGEMENTS}

The work of Francesco Marazzi was supported by the SwissEuropean Mobility Program.

\section{REFERENCES}

[1] Leahy MJ, de Mul FF, Nilsson GE, et al. Principles and practice of the laser-Doppler perfusion technique. Technol Health Care. 1999; 7(2-3): 143-62.

[2] Bonner R, Nossal R. Model for laser Doppler measurements of blood flow in tissue. Applied Optics. 15 June 1981; 20(12).

[3] Stern MD. In vivo evaluation of microcirculation by coherent light scattering. Nature. 1975; 254: 56-58. PMid:1113878. https ://do i.org/10.1038/254056a0

[4] Humeau A, Steenbergen W, Nilsson H, et al. Laser Doppler perfusion monitoring and imaging: novel approaches. Medical \& Biological Engineering \& Computing. 2007; 45(5): 421-435. PMid:17340155. https://doi.org/10.1007/s11517-007-0170-5

[5] Nillson Gert E, Anneli J, Karin W. Tissue perfusion monitoring and imaging by coherent light scattering. In: Portugal-DL tentative. International Society for Optics and Photonics. 1992. p. 90-109.

[6] Netten Paetrick M, et al. Skin microcirculation of the foot in diabetic neuropathy. Clinical Science. 1996; 91(5): 559-565. https: //doi.org/10.1042/cs0910559

[7] Rayman G, Hassan A, Tooke JE. Blood flow in the skin of the foot related to posture in diabetes mellitus. Br Med J (Clin Res Ed). 1986: 87-90.

[8] Tooke JE, et al. The effects of intravenous insulin infusion on skin microcirculatory flow in Type 1 diabetes. International Journal of Microcirculation, Clinical and Experimental/sponsored by the European Society for Microcirculation. 1984; 4(1): 69-83.

[9] Anne H, et al. Spectral components of laser Doppler flowmetry signals recorded in healthy and type 1 diabetic subjects at rest and during a local and progressive cutaneous pressure application: scalogram analyses. Physics in Medicine and Biology. 2004; 49(17): 3957. https://doi.org/10.1088/0031-9155/49/17/009

[10] Ray SA, et al. The association between laser Doppler reactive hyperaemia curves and the distribution of peripheral arterial disease.
European Journal of Vascular and Endovascular Surgery. 1999; 17(3): 245-248. PMid:10092899. https://doi.org/10.1053/ejvs. 1 998.0737

[11] Nilsson GE. Peripheral vascular diseases Laser-Doppler Blood Flowmetry ed AP Shepherd and PA Oberg. 1990: 201-213, Kluwer, Boston.

[12] Bunker CB, et al. The effects of topically applied hexyl nicotinate lotion on the cutaneous blood flow in patients with Raynaud's phenomenon. British Journal of Dermatology. 1988; 119(6): 771776. PMid:3203071. https://doi .org/10.1111/j.1365-2133. 1988.tb03502.x

[13] Lena E, et al. Afferent and efferent nerve injury in vibration white fingers. Journal of the Autonomic Nervous System. 1988; 24(3): 261266. https://doi .org/10.1016/0165-1838(88) 90126-9

[14] Wollersheim H, Reyenga J, Thien TH. Laser Doppler velocimetry of fingertips during heat provocation in normals and in patients with Raynaud's phenomenon. Scandinavian Journal of Clinical and Laboratory Investigation. 1988; 48(1): 91-95. PMid:2975399. https ://doi.org/10.3109/00365518809086618

[15] Riva CE, Harino S, Petrig BL, et al. Laser Doppler flowmetry in the optic nerve head. Exp. Eye Res. 1992; 55: 499-506. https: //doi.org/10.1016/0014-4835(92)90123-A

[16] Michelson G, Langhans MJ, Groh MJM. Clinical investigation of the combination of a scanning laser ophthalmoscope and laser Doppler flowmeter. Ger. J. Ophthalmol. 1995; 4: 342-349.

[17] Riva CE, Cranstoun SD, Grunwald JE, et al. Choroidal blood flow in the foveal region of the human ocular fundus. Invest. Ophthalmol. Visual Sci. 1994; 35: 4273-4282.

[18] Grunwald JE, Hariprasad SM, DuPont J. Effect of aging on foveolar choroidal circulation. Arch. Ophthalmol. (Chicago). 1998; 116: 150-154. https://doi .org/10.1001/archopht.116.2.150 
[19] Friedman A. A hemodynamic model of the pathogenesis of agerelated macular degeneration. Am. J. Ophthalmol. 1997; 125: 677682.

[20] Riva CE, Geiser MH, Petrig BL. Ocular blood flow assessment using continuous laser doppler flowmetry. Acta ophthalmologica. 2009.

[21] Petrig BL, Riva CE. Optic Nerve Head Laser Doppler Flowmetry: Principles and Computer Analysis. In Ocular Blood Flow, S. Karger
AG. 1996. pp. 120-127.

[22] Pemp B, Maar N, Weigert G, et al. Strategies for reducing variance in laser Doppler flowmetry measurements. 2009; 247(1): 67-71.

[23] Geiser MH, Diermann U, Riva CE. Compact laser Doppler choroidal flowmeter. Journal of Biomedical Optics. 1999: 459-464. https : //doi.org/10.1117/1.429960 